\title{
A NOTE ON THE GLOBAL STABILITY OF A SIMPLE GROWTH MODEL WITH MANY CAPITAL GOODS *
}

\author{
Edwin Burmeister, Rodney Dobell, and Kiyoshi Kuga
}

I. Introduction, 657. - II. The model, 658. - III. Reduction of the system, $660,-$ IV. The accumulation equations, 660. - V. Global stability, 660. - Appendix, 664 .

\section{INTRODUCTION}

Growth models with many assets represent an obvious advance beyond the simple one-sector model involving only a single real capital good, and permit discussion of portfolio choice, capital market trading conditions, and other important features of a general equilibrium system. One of the particularly interesting features of such models is the emergence of certain dynamic efficiency conditions, or capital market equilibrium conditions, when auxiliary variables interpreted as shadow prices of assets are introduced. These efficiency conditions, however, involve capital gains terms in a crucial way, and the behavior of asset prices may often be such that undue attention to expectations of capital gains can create unstable development. The paper of Hahn ${ }^{1}$ emphasized the way in which models having more than one capital good may in general diverge from balanced growth unless historically given asset prices may be supposed somehow to take on the uniquely correct initial values necessary to force the system to its saddlepoint equilibrium. Shell and Stiglitz ${ }^{2}$ subsequently investigated the question whether (or under what conditions) a competitive system may be presumed to have a mechanism to force asset prices to the unique values leading to steady growth equilibrium. However, it seems not to be widely realized that the "Hahn phenomenon" is not inevitable simply as a consequence of the introduction of many capital goods, but rather depends on the fact that the composition of investment is crucially influenced by anticipated capital gains. If one imagines an economy in which old capital goods are not much traded, then the instability feature emphasized by Hahn no longer need hold. This suggests that the

* Research of Burmeister was undertaken with the support of the National Science Foundation under research grant GS 1462; research of Kuga was undertaken while he held a Postdoctoral Research Fellowship at the University of Chicago. While retaining sole responsibility for all conclusions, the authors wish to acknowledge, with thanks, this support.

1. F. H. Hahn, "Equilibrium Dynamics with Heterogeneous Capital Goods," this Journal, LXXX (Nov. 1966), 633-46.

2. Karl Shell, and J. Stiglitz, "Investment Allocation in a Dynamic Economy," this Journal, LXXXI (Nov. 1967). 
one-sector Solow model ${ }^{3}$ is stable not because it has only one capital good, but rather because it has a particularly simple saving function. ${ }^{4}$ In this present note we analyze a model which is, in a sense, a multigood version of Solow's simple model. The local stability of the model was demonstrated by Burmeister and Dobell; ${ }^{5}$ the present analysis proves the global stability. ${ }^{6}$

The model supposes an institutional environment in which services of capital goods are rented, like those of labor. Each capital good is owned by a firm or firms which have essentially no opportunity to trade old capital goods, and whose earnings therefore consist only of current rentals. Of these rentals, a fraction is saved and (in the tradition of financing from retentions) invested in further equipment, and a fraction paid out to households who consume all their dividend earnings along with their wage income. While this assumed institutional environment may not be particularly plausible, it does seem true that the opportunities for trading used machines are frequently limited, and a model in which such opportunities are nonexistent may not be much worse than one in which markets for used capital goods are perfect. ${ }^{7}$ At any rate, the point of this model is that it does permit any number of different capital goods, but assumes investment decisions independent of capital gains anticipated on resale of equipment. The model is globally stable, converging to a unique balanced growth equilibrium path. In the following sections we set out the model and prove its global stability.

\section{The ModeL}

The model is standard save for its demand conditions. We have: production functions

3. R. M. Solow, "A Contribution to the Theory of Economic Growth," this Journal, (Feb. 1956), 65-94.

4. Mordecai Kurz, "The General Instability of a Class of Competitive Growth Processes," Review of Economic Studies, XXXV (April 1968), suggests a different interpretation, which is nonetheless similar to the extent that it rests on the idea of shadow prices having been "integrated out," so that capital gains no longer appear.

5. Edwin Burmeister, and Rodney Dobell, "Steady-State Behavior of Neoclassical Models with Many Capital Goods," Discussion Paper No. 72, Department of Economics, University of Pennsylvania, Dec. 1967, presented at the Econometric Society meetings, Dec. 1967.

6 . The proof of global stability given here is due to Kuga.

7. A more satisfactory model might show firms owning capital goods which are not traded speculatively while households own equity which is. Then to the extent that firms whose shares show large capital gains may be able to retain and reinvest a larger proportion of earnings, the composition of investment will be sensitive to capital gains. But whether the standard saddlepoint property at the balanced growth equilibrium will persist or not seems to be an open question. 
(1)

$$
\begin{aligned}
Y_{j}= & \mu_{j} L_{j}^{a_{j j}} K_{1 j}^{a_{1 j}} \ldots K_{n j}^{a_{n j}}(j=0,1, \ldots, n) \\
& a_{i j} \geqq 0, \mu_{j}>0, \sum_{i=0}^{n} a_{i j}=1
\end{aligned}
$$

full employment

$$
\left\{\begin{array}{l}
\sum_{j=0}^{n} L_{j}=L \\
\sum_{j=0}^{n} K_{i j}=K_{i} \quad(i=1, \ldots, n)
\end{array}\right.
$$

wage rate

$$
P_{j}\left(\partial Y_{j} / \partial L_{j}\right)=W_{0} \quad(j=0, \ldots, n)
$$

rentals

$$
P_{j}\left(\partial Y_{j} / \partial K_{i j}\right)=W_{i} \quad\left(\begin{array}{rl}
i & =1, \ldots, n ; \\
j & =0, \ldots, n)
\end{array}\right.
$$

saving and demand

$$
\begin{cases}P_{i} Y_{i}=s_{i} W_{i} K_{i} \quad(i=1, \ldots, n) & 0 \leqq s_{i} \leqq 1 \\ P_{0} Y_{0}=W_{0} L+\sum_{i=1}^{n}\left(1-s_{i}\right) W_{i} K_{i} & (i=1, \ldots, n)\end{cases}
$$

where the notation is as follows:

$Y_{j}$ denotes the output flow of the $j^{\text {th }}$ commodity, with $Y_{0}$ designating the consumption good, $Y_{1}, \ldots, Y_{n}$ the capital goods;

$L_{j}$ denotes the labor input into sector $j, j=0, \ldots, n$;

$K_{i j}$ denotes the input of service of capital good $i$ into sector $j, i=1, \ldots, n, j=0, \ldots, n$;

$L$ denotes available labor supply, assumed to grow exogenonsly at rate $g$;

$K_{i}$ denotes the quantity of the $i^{\text {th }}$ capital good, $i=1, \ldots$, $n$; the $i^{\text {th }}$ capital good is assumed to depreciate at the constant (exponential) rate $\delta_{i}$;

$P_{j}$ denotes the price of the $j^{\text {th }}$ commodity;

$W_{o}$ denotes the nominal wage rate;

$W_{i}$ denotes the nominal rental rate for the $i^{\text {th }} \operatorname{good}, i=1$, ..., $n$

$s_{i}$ denotes the constant saving rate, $0 \leqq s_{i} \leqq 1$, adopted by firms owning capital good $i, i=1, \ldots, n$.

Equation (1) expresses the assumption that all production functions are Cobb-Douglas; in addition we assume:

AI. Labor is required, directly or indirectly, to produce a positive quantity of any commodity.

Thus equations (1)-(5) together express the temporary equili- 
brium at any moment $t$, when all stocks of capital goods and labor are given.

\section{Reduction of the System}

After tedious substitution, invoking Assumption AI, the system (1)-(5) may be written in an intensive form, as a function of per capita factor endowments alone:

$$
Y_{j} / L=y_{j}=\xi_{j} k_{1}^{a_{1 j}} \ldots k_{n}^{a_{n j}} \quad(j=0, \ldots, n)
$$

where $k_{j}=K_{j} / L$ and $\xi_{j}$ is a positive constant. The Appendix sketches the derivation of equation (6).

\section{The Accumulation Equations}

Supplementing (6) with the usual growth equations

$$
D k_{i}=y_{i}-\left(g+\delta_{i}\right) k_{i} \quad(i=1, \ldots, n)
$$

(where the notation $D x$ denotes the time derivative of $x$ ), the model is expressed as a causal system which determines the growth and evolution of this economy over time. From (7) it is straightforward to calculate an equilibrium configuration $\left(k_{1}^{*}, \ldots, k_{n}^{*}\right)$ and to show that it is unique. Introducing

$$
z_{i}=k_{i} / k_{i}^{*} \quad(i=1, \ldots, n)
$$

equations (6) and (7) may be combined and written as

$$
D z_{i}=\gamma_{i}\left[z_{1}^{a_{1 i}} \ldots z_{n}^{a_{n i}}-z_{i}\right] \quad(i=1, \ldots, n)
$$

where $\gamma_{i}=g+\delta_{i}$.

\section{Global Stability}

We may now state the theorem: ${ }^{8}$

Theorem: The balanced growth path of the model (1)-(5) is globally stable, that is, any solution $z(t)$ of (9) starting from any positive initial value $z(0)>0$ tends to the unique equilibrium configuration $z^{*}=(1, \ldots, 1)^{\prime}$ as $t$ tends to infinity.

Proof: We prove this result in several steps.

8. It is perhaps worthwhile to point out that the homogeneous causal system written in terms of $L$ and $K_{\mathfrak{t}}$, rather than the intensive variables $k_{\mathfrak{l}}$, is a decomposable system, so that the theorems stated by Michio Morishima, Equilibrium, Stability and Growth (Oxford, England: Clarendon Press, 1964) and R. M. Solow and P. A. Samuelson "Balanced Growth under Constant Returns to Scale," Econometrica, Vol. 21 (July 1953), 412-24, cannot be directly applied. 
1. Introducing the functions

$$
f_{i}=\gamma_{i}\left(z_{1}^{a_{1 i}} \ldots z_{n}^{a_{n i}}-\sum_{1}^{n} a_{j i} z_{j}\right) \quad(i=1, \ldots, n)
$$

one may write $(9)$ in a standard form as

$$
D z=\Gamma\left(a^{\prime}-I\right) z+f
$$

where

$$
\begin{aligned}
& z=\left[\begin{array}{c}
z_{1} \\
\cdot \\
z_{n}
\end{array}\right], \quad f=\left[\begin{array}{c}
f_{1} \\
\cdot \\
\cdot \\
f_{n}
\end{array}\right] \\
& a=\left[\begin{array}{ccc}
a_{11} & \ldots & a_{1 n} \\
\cdot & & \cdot \\
\cdot & & \cdot \\
a_{n 1} & \ldots & a_{n n}
\end{array}\right] \quad \Gamma=\left[\begin{array}{llll}
\gamma_{1} & & & 0 \\
& \cdot & & \\
& & \cdot & \\
0 & & & \gamma_{n}
\end{array}\right]
\end{aligned}
$$

and where $a^{\prime}$ denotes the transpose of the matrix $a$. The solution to equation (11) may be written ${ }^{9}$

$$
z=u+\int_{0}^{t} U\left(t-t_{1}\right) f\left(t_{1}\right) d t_{1}
$$

where $u$ is the solution of the vector differential equation

$$
D u=\Gamma\left(a^{\prime}-I\right) u, \quad u(0)=z(0)
$$

and $U$ is the solution of the matrix differential equation

$$
D U=\Gamma\left(a^{\prime}-I\right) U \quad U(0)=I .
$$

2. Let us now note the following facts.

(i) The real part of any characteristic value of the matrix $\left(a^{\prime}-I\right)$ is negative, and hence so is that of any characteristic value of the matrix $\Gamma\left(a^{\prime}-I\right)$. This implies that the solution $u(t)$ to equation (13) tends to zero as $t$ tends to infinity.

(ii) The matrix $U(t)=\exp \left\{\Gamma\left(a^{\prime}-I\right) t\right\} \geqq 0$. In fact, from equation (14), the $i j^{\text {th }}$ element of $U$ satisfies $D U_{i j}(t)=\gamma_{i} \sum_{k=1}^{n}\left(a_{k i}-\delta_{k i}\right) U_{k j}$, and it is clear that if $U_{i j}$ becomes zero, $D U_{i j}(t) \geqq 0$. The solution $U(t)$ to (14) also tends to zero as $t$ goes to infinity.

(iii) For $\beta_{i}>0, \stackrel{n}{\Sigma} \beta_{i}=1, \quad x_{i} \geqq 0$, one has ${ }^{1}$

9. See Theorem 4, Richard Bellman, Stability Theory of Differential Equations (New York: McGraw-Hill, 1953), p. 14.

1. Edwin F. Beckenbach, and Richard Bellman, Inequalities (New York: Springer Verlag, 1965), p. 13. 


$$
\prod_{i=1}^{n} X_{i}^{\beta_{i}} \leqq \sum_{i=1}^{n} \beta_{i} x_{i} .
$$

Hence

$$
f_{i}(t) \leqq a_{o i} \gamma_{i} \quad(i=1, \ldots, n) .
$$

(iv) $\left[\exp \left\{\Gamma\left(a^{\prime}-I\right) t\right\}\right]\left[\Gamma\left(a^{\prime}-I\right)\right]^{-1}$

$$
\begin{aligned}
& =\sum_{i=0}^{\infty} \frac{\left[\Gamma\left(a^{\prime}-I\right)\right]^{i}}{i !} t^{i}\left[\Gamma\left(a^{\prime}-I\right)\right]^{-1} \\
& =\left[\Gamma\left(a^{\prime}-I\right)\right]^{-1}\left[\exp \left\{\Gamma\left(a^{\prime}-I\right) t\right\}\right] .
\end{aligned}
$$

(v) From the identity $-\left(a^{\prime}-I\right)^{-1}\left(a^{\prime}-I\right)=-I$ we may obtain, upon premultiplying both sides into the column vector $(1,1, \ldots, 1)^{\prime}$, the result

$$
-\left(a^{\prime}-I\right)^{-1} a_{0}^{\prime}=(1 \ldots 1)^{\prime} \text {. }
$$

Hence

where

$$
-\left[\Gamma\left(a^{\prime}-I\right)\right]^{-1} f^{*}=(1, \ldots, 1)^{\prime}
$$

$$
f^{*}=\left[a_{o 1} \gamma_{1}, \ldots, a_{o n} \gamma_{n}\right]^{\prime} .
$$

3. Now let us evaluate the solution $z(t)$ given in (12).

$$
\begin{aligned}
& z=u+\int_{0}^{t} U\left(t-t_{1}\right) f\left(t_{1}\right) d t_{1} \\
& \leqq u+\left(\int_{0}^{t} U\left(t-t_{1}\right) d t_{1}\right) \cdot f^{*} \quad \text { (using (ii) and (iii)) } \\
& =u+\left(\int_{0}^{t}\left\{\exp \Gamma\left(a^{\prime}-I\right)\left(t-t_{1}\right)\right\} d t_{1}\right) \cdot f^{*} \\
& =u+\left(\exp \Gamma\left(a^{\prime}-I\right) t\right) \text {. } \\
& \left(\int_{0} \exp \left\{-\Gamma\left(a^{\prime}-I\right) t_{1}\right\} d t_{1}\right) \cdot f^{*} \\
& \begin{array}{c}
=u-\left(\exp \Gamma\left(a^{\prime}-I\right) t\right) \cdot\left\{\Gamma\left(a^{\prime}-I\right)\right\}^{-1} . \\
\left(\exp \left\{-\Gamma\left(a^{\prime}-I\right) t\right\}-I\right) \cdot f^{*}
\end{array} \\
& \begin{aligned}
= & (1, \ldots, 1)^{\prime}+u+\left(\exp \left(\Gamma\left(a^{\prime}-I\right) t\right)\right) \\
& \left(\Gamma\left(a^{\prime}-I\right)\right)^{-1} \cdot f^{*} \quad \text { (using (iv) and (v)). }
\end{aligned}
\end{aligned}
$$

4. We can establish a simple inequality useful in providing the theorem.

\section{Lemma: ${ }^{2}$}

For $a_{i} \geqq 1, a_{k} \leqq 0(k \neq i), \sum_{\zeta=1}^{n} a_{\zeta}=1, x_{\zeta}>0(\zeta=1,2, \ldots, n)$, $n \geqq 2$, one has the inequality

$$
\prod_{j=1}^{n} x_{j}^{a_{j}} \geqq \sum_{j=1}^{n} a_{j} x_{j}
$$

2. Global stability for the case $n=1$ being obvious, we need concern ourselves here only with the case $n>1$. 
Proof: We start with the established inequality, ${ }^{3}$

$$
x^{a}-a x+a-1 \geqq 0
$$

for

$$
x>0, \quad a \leqq 0 .
$$

First using (17), the validity of (16) is proved for the case $n=2$. If we put $x=x_{1} / x_{2}$ in (17), then we get

$$
x_{1} x_{2}^{1-a} \geqq a x_{1}+(1-a) x_{2} \quad \text { for } a \leqq 0 .
$$

Suppose (16) is true for $n$, and put

$$
\begin{aligned}
& x_{j}=x_{j}^{*}, \beta_{j}=a_{j} \quad(j=1,2, \ldots, n-1) \\
& x_{n}^{*}=x_{n}^{a_{n} / \beta_{n}} x_{n+1}^{a_{n+1} / \beta_{n}}, \quad \beta_{n}=a_{n}+a_{n+1}<0,
\end{aligned}
$$

where, by suitable renumbering, we may consider $a_{\imath} \geqq 1$ $a_{j} \leqq 0, i \neq n, i \neq n+1, j \neq 1$. Then

$$
\begin{aligned}
& \prod_{k=1}^{n+1} x_{k}=\prod_{k=1}^{n}\left(x_{k}^{*}\right)^{\beta_{k}} \\
& \geqq \sum_{k=1}^{n} \beta_{k} x_{k}^{*} \quad \text { (by induction) } \\
& =\sum_{k=1}^{n-1} a_{k} x_{k}+\left(a_{n}+a_{n+1}\right)\left(x_{n}^{a_{n} / \beta_{n}} x_{n+1}^{a_{n+1} / \beta_{n}}\right) \\
& \geqq \sum_{k=1}^{n+1} a_{k} x_{k} . \quad \text { (using } a_{n}+a_{n+1}<0, \text { and (iii)). }
\end{aligned}
$$

5. Let us rewrite $(9)$ by putting $v_{i}=1 / z_{i}(i=1,2, \ldots, n)$, thus obtaining

$$
D v_{i}=-\gamma_{i}\left(v_{1}-a_{14} \ldots v_{i}-a_{i 4}+2 \ldots v_{n}^{-a_{n i}-v_{i}}\right)(i=1,2, \ldots, n) .
$$

Let us put

$$
\begin{array}{r}
q_{i}=\gamma_{i}\left(v_{1}-a_{14} \ldots v_{i}-a_{i t}+2 \ldots v_{n}-a_{n i}-\sum_{j=1}^{n}\left(-a_{j i}+2 \delta_{j i}\right) v_{j}\right) \\
(i=1,2, \ldots, n) .
\end{array}
$$

Then by the lemma, we have

(20) $\quad q_{i} \geqq-\gamma_{i} a_{o i}$.

Substituting (19) into (18), we get a differential equation similar to (11),

$$
D v=\Gamma\left(a^{\prime}-I\right) v-q
$$

where

3. Beckenbach, op. cit., p. 12.

4. In $(9)$, it is easy to see that $z(t)>0$, if $z(0)>0$. In fact, since $\min$ $z_{i}(0) \leqq z_{j}(t) \leqq \max \quad z_{i}(0), \quad(j=1,2, \ldots, n), \quad z_{1}{ }^{a_{1 i}} \ldots z_{n}{ }^{a_{k}}$ is bounded. Therefore $D z_{i} / z_{i}$ becomes positive before $z_{i}$ can approach zero. 


$$
v=\left[\begin{array}{c}
v_{1} \\
\cdot \\
\dot{v_{n}}
\end{array}\right],
$$$$
q=\left[\begin{array}{c}
q_{1} \\
\cdot \\
\dot{q_{n}}
\end{array}\right] .
$$

Analogously to step 3, we may write

$$
v \leqq(1, \ldots, 1)^{\prime}+u+\left(\exp \Gamma\left(a^{\prime}-I\right) t\right) \cdot\left(\Gamma\left(a^{\prime}-I\right)\right)^{-1} \cdot f^{*} .
$$

Combining (15) and (22), we have

$$
1 / \phi_{i}(t) \leqq z_{i}(t) \leqq \phi_{i}(t) \quad(i=1,2, \ldots, n)
$$

where $\phi_{i}(t)$ is the $i^{\text {th }}$ element of the right-hand side of (15). Since $\phi_{i}(t)$ tends to unity as $t$ tends to infinity, all $z_{i}(t)$ tend to 1 , by (23). This proves the theorem.

\section{APPENDIX}

The reduction of the momentary equilibrium to the equations (6) is described in Burmeister and Dobell. ${ }^{5}$ The necessary steps may be sketched as follows: Using (1), (3), (4), and (5), the full employment equations (2) can be rearranged as

$$
h=[I-B]^{-1} \cdot b \text {, }
$$

$$
h=\left[\begin{array}{c}
\frac{k_{1} W_{1}}{W_{0}} \\
\cdot \\
k_{n} \dot{W}_{n} \\
W_{0}
\end{array}\right], b=\left[\begin{array}{c}
a_{10} \\
\cdot \\
\cdot \\
a_{n o}
\end{array}\right], B=\left[\begin{array}{cc}
\left(1-s_{1}\right) a_{10}+a_{11} s_{1} \ldots\left(1-s_{n}\right) a_{10}+a_{1 n} s_{n} \\
\cdot \\
\cdot \\
\left(1-s_{1}\right) a_{n o}+a_{n 1} s_{1} \ldots\left(1-s_{n}\right) a_{n o}+a_{n n} s_{n}
\end{array}\right] .
$$

Assumption AI implies $[I-B]^{-1} \cdot b>0$, and therefore from (24) we may put

$$
k_{i} W_{i} / W_{o}=\zeta_{i}>0 \quad(i=1,2, \ldots, n)
$$

where

$$
\left[\begin{array}{c}
\zeta_{1} \\
\cdot \\
\dot{\zeta_{n}}
\end{array}\right]=(I-B)^{-1} \cdot b .
$$

On the other hand, the marginal conditions (3) and (4) are reduced to

5. Op. cit. 
(26)

$$
p_{j} / W_{j}=\eta_{j}\left(W_{1} / W_{o}\right) a_{1 j} \ldots\left(W_{j} / W_{o}\right)^{a_{j j}-1} \ldots\left(\begin{array}{c}
\left(W_{n} / W_{o}\right) a_{n j} \\
(j=1,2, \ldots, n)
\end{array}\right.
$$

where

$$
\eta_{j}=1 /\left(a_{o j} a_{o j a_{1 j}} a_{1 j} \ldots a_{n j} a_{n j} \mu_{j}\right) .
$$

Substituting (25) and (26) into (5), we get (6) and

$$
\xi_{j}=\frac{s_{j}}{n_{j}} \zeta_{1}-a_{1 j} \ldots \zeta_{j}-a_{j j}+1 \ldots \zeta_{n}-a_{n j}(j=1,2, \ldots, n) .
$$

University of Pennsylvania

HARVARD UNIVERSITY

The University of Chicago AND OsaKa UNIVERSITY 